\title{
Objective Evaluation of Olfactory and Taste Dysfunction Among COVID-19 Patients: A Cross Sectional Study from Tribal India
}

\author{
Izhar Khan ${ }^{1} \cdot$ Vikas Gupta $^{2}$ (1) $\cdot$ Sanjay Kumar Shukla ${ }^{1}$
}

Received: 29 July 2021/ Accepted: 2 October 2021 / Published online: 19 October 2021

(C) Association of Otolaryngologists of India 2021

\begin{abstract}
There is a varying prevalence of olfactory and taste dysfunction (OTD) in COVID-19 patients, with a higher prevalence reported in the European population as compared to the Asian population. Psychophysical tests are crucial to determine the exact frequency, extent and clinical characteristics of these OTDs. The present study objectively evaluated the OTD for patients treated in the Dedicated COVID-19 Hospital (DCH) in Shahdol. This prospective cross-sectional study was conducted after IEC approval in DCH Shahdol for a period of four months among RT-PCR positive patients, and they were evaluated using validated chemosensitive psychophysical test during ENT consultation to identify OTD. The sample size was calculated as 92 considering prevalence (p) of OTD as $41.3 \%$ by applying formula: $n=\left(\mathrm{Z}^{1-\mathrm{a} / 2}\right)^{2} \times p(1-p) / d^{2}$. The information pertaining to the subjects was kept anonymous and confidential. During data analysis, an association was significant for $p$ value $<0.05$. It was observed that $45.5 \%$ of subjects reported either loss of taste or smell. During objective evaluation, olfactory and taste dysfunction was observed among $42.4 \%$ of subjects (95/ 224). The Chi-square analysis reflected statistically significant difference $(p<0.05)$ between subjects with OTD
\end{abstract}

Vikas Gupta

drvikasgupta1988@gmail.com

Izhar Khan

kmdizhar@gmail.com

Sanjay Kumar Shukla

sanjaykumarshuklams@gmail.com

1 Department of Otorhinolaryngology, Government Medical College, Shahdol, Madhya Pradesh, India

2 Department of Community Medicine, Government Medical College, Shahdol, Madhya Pradesh, India and without OTD for the variables such as days from onset of symptoms to admission, and symptoms (fever, sore throat and shortness of breath). Taste and smell are among the important senses and in India they are mostly subjectively evaluated for COVID-19 induced OTD which results in underreporting of these symptoms. So, a gold standard objective evaluation should be taken into consideration to evaluate OTD.

Keywords Cycle threshold $(\mathrm{Ct})$ value . Chemosensory loss · Nasopharyngeal swab · Anosmia . Ageusia

\section{Introduction}

An unprecedented disease hit the world some time ago. The World Health Organization (WHO) declared this a corona virus disease-19 (COVID-19) pandemic on 11th March 2020. It has affected over 103,989,900 and killed 2,260,259 people by 05/02/2021, where patients suffering from the COVID-19 present with a wide array of symptoms ranging from fever and respiratory distress to gastrointestinal symptoms [1]. The death count being rapidly rising, though the level $(5.6 \%)$ has not reached the infliction caused by the other members of the coronavirus family such as SARS (13\%) and MERS (35\%) [2].

However, COVID is far more transmissible with an estimated reproduction number (R0) 3.32 [3]. Now, India too, is in the grip of the pandemic and, in terms of absolute numbers, the 2nd worst affected country after USA. Early countrywide lockdown helped in delaying the spread and shift the peak, and gave time to create infrastructure to face the surge. Despite this, 10,790,183 cases and 154,703 deaths have been reported in India as of 05/02/2021 [4]. 
Many professional organizations have now recognized olfactory and taste dysfunction (OTD) as one of symptoms of COVID-19 and included them in their diagnostic guidelines. There is a varying prevalence of olfactory and taste dysfunction in COVID-19 patients, with a higher prevalence reported in the European population as compared to the Asian population [5]. Due to the low sensitivity and specificity of listed symptoms of COVID-19 by Centres for Disease Control and Prevention (CDC), United States, there is need for more data on potentially more specific symptoms such as loss of sense of smell as specific symptoms like loss of smell and taste are common with other neurotropic viral infections [6, 7].

Psychophysical tests are crucial to determine the exact frequency, extent and clinical characteristics of these chemosensitive disorders, and to monitor their recovery over time. However, most of the studies currently available in the Indian literature are based on anamnestic or observational evidence [8]. So, the purpose of the present study was to objectively evaluate the olfactory and taste dysfunction, using psycho-physiological objective tests, among COVID-19 patients treated in the Dedicated COVID-19 Hospital (DCH) in Shahdol. This will enable us to identify chemosensitive symptoms that would have earlier been missed and to provide effective management of cases with optimal efficiency and better outcomes for the future upcoming third COVID-19 wave.

\section{Materials and Methods}

\section{Study Setting and Design}

This prospective cross-sectional study was conducted in Dedicated COVID-19 Hospital (DCH) at Government Medical College, Shahdol, Madhya Pradesh for a period of four months (March 2021 to June 2021). GMC Shahdol was recognized as official site as $\mathrm{DCH}$ for managing COVID-19 patients on 27/03/2020 when the disease started to occur in the epidemic proportion in India.

\section{Study Population and Sample Size}

The study subjects were the patients (18 years or above) tested positive $(\mathrm{Ct}$ value less than 35 was considered as COVID-19 positive) for COVID-19 infection by reverse transcription- polymerase chain reaction (RT-PCR) based test with mild, moderate and severe disease admitted to DCH that is designated as COVID-19 hospitals by the State Government. The study did not included children ( $<18$ years of age), patients with psychiatric or neurological disorders, patients with history of previous surgery or radiation in the oral and nasal cavities, chronic rhinosinusitis and; pre-existing smell and taste disturbances.

The sample size was calculated $(n=92)$ considering the proportion of COVID-19 positive patients having OTD on objective evaluation as $41.3 \%$ (Rajkumar et al.) with confidence level of $95 \%$ and $10 \%$ absolute allowable error by applying the following formula: $n=\left(Z^{1-\mathrm{a} / 2}\right)^{2} \times p(1-p) /$ $d^{2}$; where $\mathrm{Z}=$ Standard normal variate for level of significance [at $5 \%$ type $\mathrm{I}$ error $(P<0.05), \mathrm{Z}=1.96$ for 2 -sided test], $\mathrm{a}=$ Level of significance $(0.05), \quad p=$ Prevalence (proportion- 50\%), d = Absolute Allowable error $(10 \%)$, $n=$ Sample Size, so all eligible study participants which counted to be 224 were included in the study using convenient sampling method (Fig. 1) [9].

\section{Data Collection}

Participation in this survey was voluntary and was not compensated. After obtaining approval to conduct study from the Institutional Review Board (IERB), the admitted COVID-19 patients were approached with an introductory paragraph outlining the aims and objectives of the study. Informed consent was obtained from eligible subjects prior to participation. The demographic details (age and gender) and detailed clinical history (symptoms, co-morbidities, admitted to HDU/Isolation/ICU) the patient was taken.

The chemosensitive psychophysical test procedure was explained to the subjects and both the olfactory and gustatory functions were objectively evaluated during ENT consultation (Fig. 2). Olfactory function assessment was carried out by means of the Connecticut Chemosensory Clinical Research Center orthonasal olfaction test (CCCRC) [10]. The CCCRC is a simple, validated, and widely used test (Fig. 2). The threshold and identification test scores, after being analyzed separately, were finally converted into the CCCRC composite score (Normal $=90-100$, Mild hyposmia $=70-80$, Moderate hyposmia $=50-60, \quad$ Severe $\quad$ hyposmia $=20-40 \quad$ and Anosmia $=0-10$ ).

A standardized and validated test, which investigates the ability to perceive four primary tastes (sweet, salty, sour, and bitter) was used to evaluate the taste function (Fig. 2). Taste score ranged from 0 to 4 allowing to classify the patients into four categories (Normal $=4$, mild hypogeusia $=3$, moderate hypogeusia $=2$, severe hypogeusia $=1$, and ageusia $=0$ ). The information pertaining to subjects was kept anonymous and confidential [11].

\section{Data Analysis}

Collected data was entered in the MS Excel spreadsheet, was coded appropriately and was later cleaned for any possible errors. Analysis was carried out using IBM SPSS 
Fig. 1 Consolidated Standards of Reporting Trials (CONSORT) flowsheet

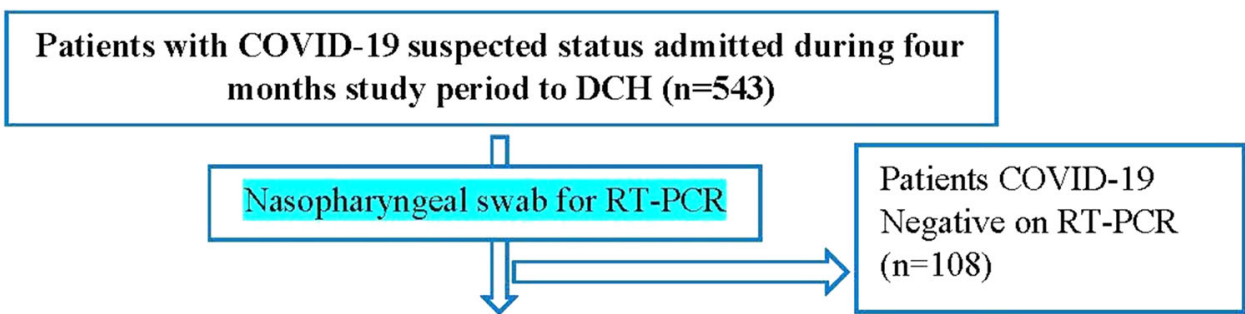

COVID-19 positive patients with mild, moderate and severe symptoms $(n=435)$

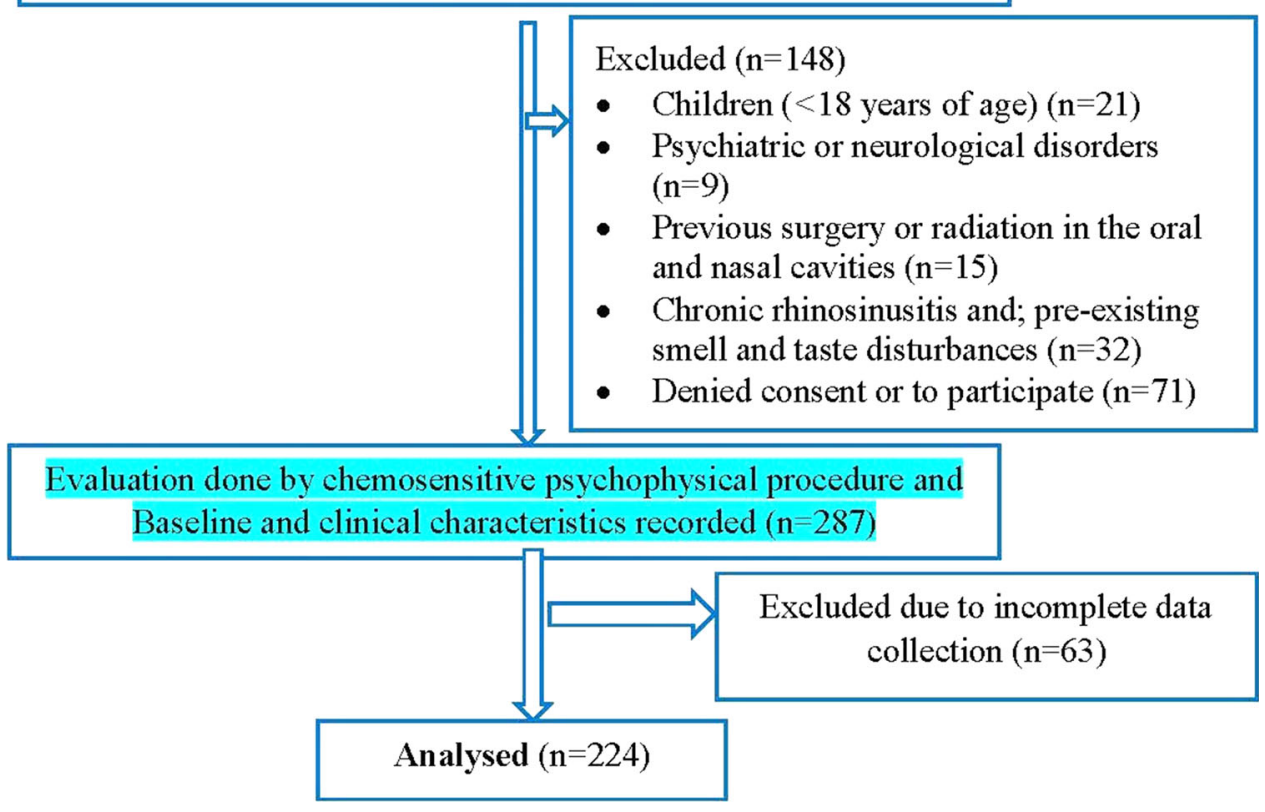

Statistics for Windows, Version 22.0 (IBM Corp. Armonk, NY, USA). During data cleaning, to facilitate association of variables, more variables were created. Clear values for various outcomes were determined before running frequency tests. Categorical data was presented as percentages (\%) and quantitative data was presented as mean (standard deviation). Chi-square was used as test of significance to observe the difference between categorical variables in two groups (with OTD and without OTD). All tests (two tailed) were performed at a 5\% level of significance; thus, an association was significant if the $\mathrm{p}$ value was less than 0.05 .

\section{Ethical Consideration}

All ethical issues were followed during the study. Participation was voluntary and participants were allowed to withdraw from study at any moment. No personal data was recorded. Participants were assured that all data collected was used only for the current study. Study was initiated after approval from IERB (Project ID: IERC/21/03/004). Additionally, before filling the questionnaire, participants were asked to give their consent to participate in the study.

\section{Results}

The present study included 224 subjects and the mean age of the subjects was $35.4 \pm 15.5$ years. As shown in Table 1, there was nearly equal presentation of subjects from various age groups with maximum participation from younger subjects $(<30$ years, 61/224, 27.2\%) and least from aged ones ( $>50$ years, 48/224, 21.5\%). The subjects enrolled in the study were mostly the males $(54.9 \%$, 123/224). The comorbid conditions such as hypertension, diabetes mellitus, asthma, COPD and others were reported among nearly one third of subjects $(28.6 \%, 64 / 224)$. The history of alcohol intake, smoking or tobacco chewing was observed among one fifth of enrolled subjects (20.9\%, 47/224).

Table 2. shows that only $65.2 \%$ (146/224) of enrolled subjects got admitted to hospital within 5 days of onset of symptoms. Around four fifth of subjects $(80.3 \%, 180 / 224)$ reported history of fever or malaise followed by cough among $34.6 \%$ of subjects (77/224). Only $5.8 \%$ of subjects (13/224) required mechanical ventilation as $\mathrm{O}_{2}$ support. The reported loss of taste or smell was observed among 


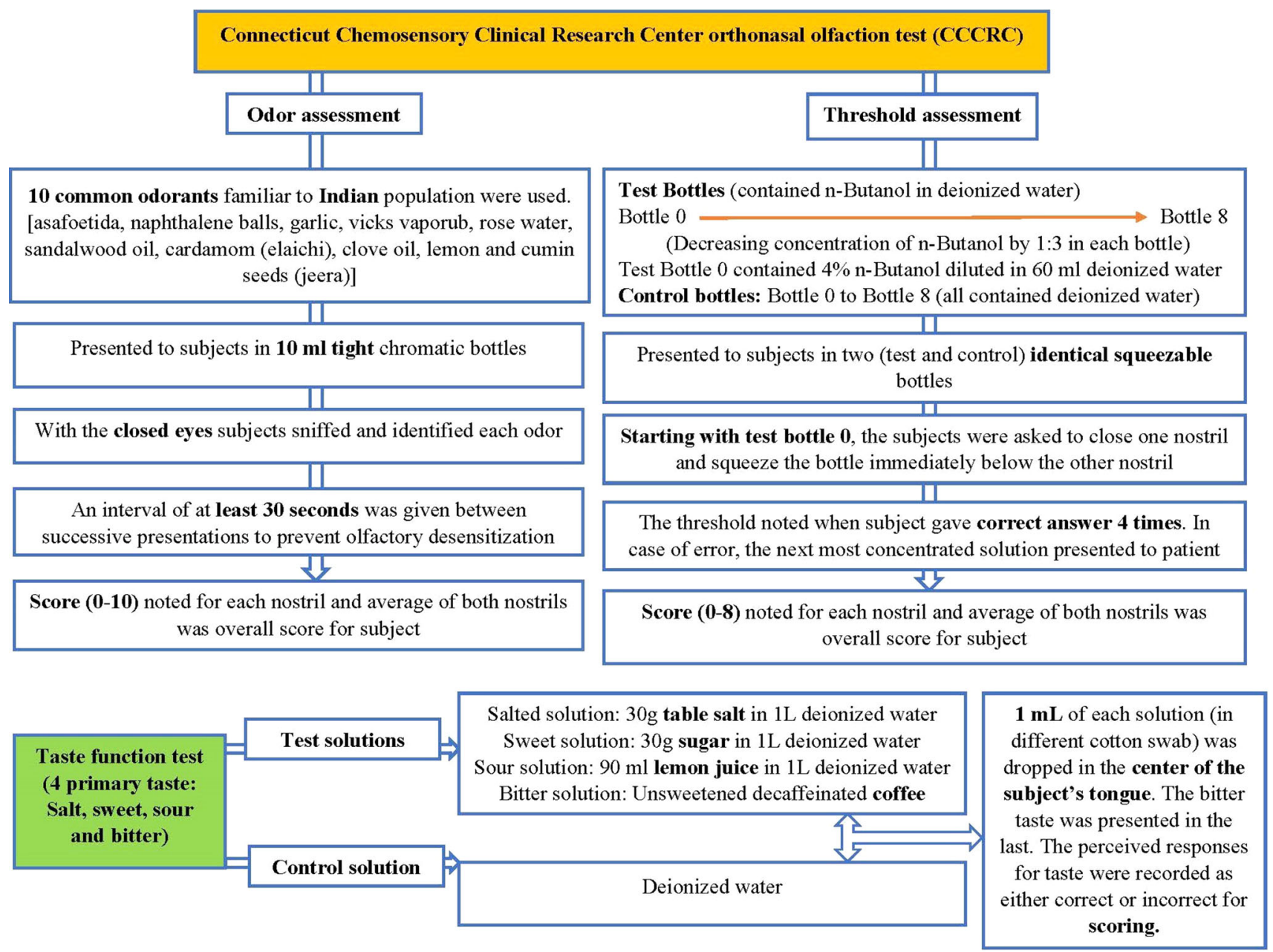

Fig. 2 Chemosensitive psychophysical test procedure for olfactory and gustatory dysfunctions among subjects

Table 1 Baseline characteristics of study subjects $(N=224)$

\begin{tabular}{lc}
\hline Variables & Number $(\%) / \mathrm{Mean} \pm \mathrm{SD}$ \\
\hline Mean age & $35.4 \pm 15.5$ \\
Age group & $61(27.2)$ \\
$\quad<30$ years & $59(26.3)$ \\
$30-40$ years & $56(25.0)$ \\
$41-50$ years & $48(21.5)$ \\
$>50$ years & $123(54.9)$ \\
Gender & $101(45.1)$ \\
Male & \\
Female & $47(20.9)$ \\
Tobacco/Alcohol intake & $177(79.1)$ \\
Yes & \\
No & \\
Any comorbidities & $64(28.6)$ \\
Yes & $160(71.4)$ \\
No & \\
\hline
\end{tabular}


Table 2 Clinical characteristics of study subjects $(N=224)$

\begin{tabular}{|c|c|}
\hline Variables & Number $(\%)$ \\
\hline \multicolumn{2}{|c|}{ Days from onset of symptoms to admission } \\
\hline$<5$ days & $146(65.2)$ \\
\hline $5-10$ days & $35(15.6)$ \\
\hline$>10$ days & $43(19.2)$ \\
\hline \multicolumn{2}{|l|}{ Symptoms* } \\
\hline Fever/Malaise & $180(80.3)$ \\
\hline Shortness of breath & $59(26.3)$ \\
\hline Cough & $77(34.6)$ \\
\hline Sore throat & $42(18.8)$ \\
\hline Nasal discharge & $39(17.4)$ \\
\hline \multicolumn{2}{|l|}{ Reported loss of smell/taste } \\
\hline Both loss of smell and taste & $57(25.4)$ \\
\hline Only loss of smell & $24(10.7)$ \\
\hline Only loss of taste & $21(9.4)$ \\
\hline Total & $102(45.5)$ \\
\hline Neither loss of taste nor loss of smell & $122(54.5)$ \\
\hline \multicolumn{2}{|c|}{ Loss of smell/taste from clinical onset of symptoms $(n=102)$} \\
\hline$<5$ days & $58(56.8)$ \\
\hline 5 days or more & $44(43.2)$ \\
\hline \multicolumn{2}{|l|}{ Admitted in } \\
\hline HDU/Private ward & $193(86.2)$ \\
\hline ICU & $31(13.8)$ \\
\hline \multicolumn{2}{|l|}{ Oxygen support } \\
\hline $\mathrm{O}_{2}$ port or concentrator & $36(16.1)$ \\
\hline Mechanical & $13(5.8)$ \\
\hline None & $175(78.1)$ \\
\hline
\end{tabular}

*Multiple responses

$45.5 \%$ of study subjects (102/224) and among them 9.4\% of subjects reported only loss of taste.

The present study showed that loss of taste and smell were among the early occurring symptoms in COVID-19 subjects, as $56.8 \%$ of subjects (58/102) reported loss of taste or smell occurring within the first 5 days of the clinical onset. Also, among $10.7 \%$ of study subjects (24/ 224), loss of taste and smell was among the first clinical manifestation of the COVID-19, and 7.1\% of subjects (16/ 224) had loss of taste and smell that was sudden in onset along with no nasal obstruction or rhinitis symptoms.

Table 3. shows that olfactory and gustatory functions evaluated objectively during ENT consultation revealed $4.0 \%$ of subjects $(9 / 224)$ were having ageusia and $5.4 \%$ of subjects $(12 / 224)$ were having anosmia. Overall, olfactory and taste dysfunction (OTD) was observed among $42.4 \%$ of subjects $(95 / 224)$.

In the present study, during the hospital stay, 53.9\% (55/ 102) of subjects expressed that the they have recovered from the symptoms of loss of taste and smell, but when they were objectively evaluated it was observed that $70.9 \%$ (39/55) of subjects still had a residual degree of loss of taste and smell.

Table 4. shows that there was no statistically significant difference $(p>0.05)$ between subjects with OTD and without OTD for the given variables such as age groups, gender, tobacco/alcohol intake and associated comorbidities. It was observed that $56.8 \%$ of subjects (54/95) with OTD, got admitted to hospital within 5 days of onset of symptoms whereas; $71.3 \%$ of subjects (92/129) without OTD, got admitted to hospital within 5 days of onset of symptoms. The Chi-square analysis reflected statistically significant difference $(p<0.05)$ among subjects with OTD and without OTD for the variables such as days from onset of symptoms to admission, and symptoms (fever, sore throat and shortness of breath). 
Table 3 Distribution of study subjects based on chemosensitive psychophysical test $(N=224)$

\begin{tabular}{ll}
\hline Variables & Number $(\%)$ \\
\hline Taste dysfunctions & $147(65.6)$ \\
Normal & $36(16.1)$ \\
Mild hypogeusia & $18(8.0)$ \\
Moderate hypogeusia & $14(6.3)$ \\
Severe hypogeusia & $9(4.0)$ \\
Ageusia & $142(63.4)$ \\
Olfactory dysfunctions & $39(17.4)$ \\
Normal & $18(8.0)$ \\
Mild hyposmia & $13(5.8)$ \\
Moderate hyposmia & $12(5.4)$ \\
Severe hyposmia & \\
Anosmia & $64(28.6)$ \\
Olfactory and taste dysfunctions & $12(5.4)$ \\
Both Olfactory and taste dysfunctions & $19(8.4)$ \\
Only taste dysfunctions & $95(42.4)$ \\
Only Olfactory dysfunctions & $129(57.6)$ \\
Overall olfactory and taste dysfunctions & \\
Neither Olfactory nor taste dysfunctions & \\
\hline
\end{tabular}

\section{Discussion}

Sudden onset of loss of smell and taste are now well documented symptoms of COVID-19 and now have been incorporated in various diagnostic guidelines. Study by Leurs et al., showed that reduction in olfaction occurred in $74 \%$ of COVID-19 patients, while reduced sense of taste was observed in $69 \%$ of COVID-19 patients [12]. Study by Roland et al., showed that the change in smell or taste is a strong predictor for a COVID-19-positivity on RT-PCR test results and while considering the presence of change in smell or taste along with the fever, this parsimonious classifier correctly predicts $75 \%$ of COVID-19 test results [13]. Similarly, a study by De Maria et al., reflected that $50.5 \%$ of patients had extensive taste disorder and had an associated self-assessed olfactory dysfunction prior to the onset of fever and other COVID-19 symptoms [14]. A meta-analysis of 10 large cohort studies of olfactory dysfunction and nine large cohort studies of taste dysfunction, showed a prevalence of $52.5 \%$ and $43.9 \%$ respectively for olfactory dysfunction and taste dysfunction [15].

The objective evaluation of COVID-19 patients is essential to quantify the exact frequency of chemosensitive disorders, obtain a standardized classification of severity, and monitor their recovery over the time. The identification and isolation of the pauci-symptomatic patients is crucial for breaking the virus chain of infection. Such health control measures will be crucial in the upcoming months to contain any new epidemic clusters. However, most of reports published so far do not evaluate quantitatively and qualitatively the extent of chemosensory disorders in COVID-19 patients. Most of the studies are based on subjective self-evaluations of the patients, report of anamnestic data, or olfactory and gustatory questionnaire completion, without any objective test or direct medical examination [16-19]. This can be explained by the emergency situation and the risk of infection while approaching these patients without proper personal protection equipment.

The most important evidence of the present study is that the taste and olfactory dysfunctions are very frequent in COVID-19 patients. In our study, $45.5 \%$ of subjects (102/ 224) reported of having one or both problems, either loss of taste or loss of smell during the course of infection. This frequency is much higher than the frequency reported in the anamnestic-observational studies done by Varghese et al., (12.0\%) and Kou et al., (27.6\%) but lower than Thakur et al., (72.0\%) study [20-22].

The pathophysiology mechanism of olfactory and taste dysfunction in COVID-19 is still unknown. The most likely mechanism is that SARS-CoV-2 targets the angiotensinconverting enzyme 2 (ACE2) receptors found on the sustentacular and basal cells of the nasal epithelium, including the olfactory epithelium. It is also postulated that the virus invades the central nervous system through the olfactory bulb [23, 24]. So, acquisition of objective data is fundamental to understand mechanisms of olfactory and taste 
Table 4 Association between OTD and baseline/clinical characteristics of study subjects $(N=224)$

\begin{tabular}{|c|c|c|c|}
\hline \multirow[t]{2}{*}{ Variables } & \multicolumn{2}{|l|}{ OTD } & \multirow[t]{2}{*}{ Test of significance } \\
\hline & $\begin{array}{l}\text { Yes }(n=95) \\
\text { Number }(\%)\end{array}$ & No $(n=129)$ & \\
\hline \multicolumn{4}{|l|}{ Age group } \\
\hline$<30$ years $(n=61)$ & $27(28.4)$ & $34(26.4)$ & \multirow[t]{4}{*}{$\chi^{2}=1.002, \mathrm{df}=3, p=0.800$} \\
\hline $30-40$ years $(n=59)$ & $24(25.3)$ & $35(27.0)$ & \\
\hline $41-50$ years $(n=56)$ & $26(27.4)$ & $30(23.3)$ & \\
\hline$>50$ years $(n=48)$ & $18(18.9)$ & $30(23.3)$ & \\
\hline \multicolumn{4}{|l|}{ Gender } \\
\hline Male $(n=123)$ & $54(56.8)$ & $69(53.5)$ & \multirow[t]{2}{*}{$\chi 2=0.248, \mathrm{df}=1, p=0.618$} \\
\hline Female $(n=101)$ & $41(43.2)$ & $60(46.5)$ & \\
\hline \multicolumn{4}{|l|}{ Tobacco/Alcohol intake } \\
\hline Yes $(n=47)$ & $17(17.9)$ & $30(23.3)$ & \multirow[t]{2}{*}{$\chi 2=0.948, \mathrm{df}=1, p=0.330$} \\
\hline No $(n=177)$ & $78(82.1)$ & $99(76.7)$ & \\
\hline \multicolumn{4}{|l|}{ Any comorbidities } \\
\hline Yes $(n=64)$ & $27(28.4)$ & $37(28.7)$ & \multirow[t]{2}{*}{$\chi^{2}=0.002, \mathrm{df}=1, p=0.965$} \\
\hline No $(n=160)$ & $68(71.6)$ & $92(71.3)$ & \\
\hline \multicolumn{4}{|c|}{ Days from onset of symptoms to admission } \\
\hline$<5$ days $(n=146)$ & $54(56.8)$ & $92(71.3)$ & \multirow[t]{3}{*}{$\chi 2=7.500, \mathrm{df}=2, p=0.023^{\#}$} \\
\hline $5-10$ days $(n=35)$ & $15(15.8)$ & $20(15.5)$ & \\
\hline$>10$ days $(n=43)$ & $26(27.4)$ & $17(13.2)$ & \\
\hline \multicolumn{4}{|l|}{ Symptoms* } \\
\hline Fever/Malaise $(n=183)$ & $85(89.4)$ & $98(75.9)$ & $\chi 2=4.966, \mathrm{df}=1, p=0.025^{\#}$ \\
\hline Shortness of breath $(n=59)$ & $18(18.9)$ & $41(31.8)$ & $\chi 2=4.645, \mathrm{df}=1, p=0.031^{\#}$ \\
\hline Cough $(n=77)$ & $31(32.6)$ & $46(35.7)$ & $\chi 2=0.222, \mathrm{df}=1, p=0.637$ \\
\hline Sore throat $(n=42)$ & $12(12.6)$ & $30(23.2)$ & $\chi 2=4.053, \mathrm{df}=1, p=0.044^{\#}$ \\
\hline Nasal discharge $(n=39)$ & $20(21.1)$ & $19(14.7)$ & $\chi 2=1.521, \mathrm{df}=1, p=0.217$ \\
\hline \multicolumn{4}{|l|}{ Admitted in } \\
\hline HDU/Private ward $(n=193)$ & $84(88.4)$ & $109(84.5)$ & \multirow[t]{2}{*}{$\chi^{2}=0.706, \mathrm{df}=1, p=0.400$} \\
\hline $\mathrm{ICU}(n=31)$ & $11(11.6)$ & $20(15.5)$ & \\
\hline \multicolumn{4}{|l|}{ Oxygen support } \\
\hline $\mathrm{O}_{2}$ port or concentrator $(n=36)$ & $12(12.6)$ & $24(18.6)$ & \multirow[t]{3}{*}{$\chi^{2}=2.470, \mathrm{df}=2, p=0.290$} \\
\hline Mechanical $(n=13)$ & $4(4.2)$ & $9(7.0)$ & \\
\hline None $(n=175)$ & $79(83.2)$ & $96(74.4)$ & \\
\hline
\end{tabular}

*Multiple responses

\#Statiscally significant

loss in COVID-19 subjects and provide new insights into aspects of viral pathogenesis. The present study reflected that loss of taste and smell were among the early occurring symptoms in COVID-19 subjects, as $56.8 \%$ of subjects (58/ 102) reported loss of taste or smell occurring within the first 5 days of the clinical onset. Also, among $10.7 \%$ of study subjects (24/224), loss of taste and smell was among the first clinical manifestation of the COVID-19. Therefore, otorhinolaryngologists and head-neck surgeons should nowadays consider COVID-19 as a differential diagnosis of non-specific chemosensitive disorders. As reflected in present study that $7.1 \%$ of subjects $(16 / 224)$ had loss of taste and smell that was sudden in onset (within 24 to $48 \mathrm{~h}$ ) along with no nasal obstruction or rhinitis symptoms are very suspect clinical features of COVID-19. During the hospital stay, $53.9 \%$ (55/102) of subjects expressed that the they have recovered from the symptoms of loss of taste and smell, but when they were objectively evaluated it was observed that $70.9 \%$ (39/55) of subjects still had a residual degree of loss of taste and smell.

Although more than half of the study subjects reported of being suffered from complete ageusia and anosmia (102/ 
$224,45.5 \%)$, these pathological conditions were detected only in few patients (ageusia 4.0\% and anosmia 5.4\%) during the chemosensitive tests. In present study, the objective evaluation was performed at an advanced period of the clinical course with an average of around 12.3 days from the onset of clinical symptoms. An earlier evaluation would be very useful to study the olfactory and gustatory functions when they are mostly affected, but logically it is difficult as the patients in the first few days before the diagnosis are uncommonly hospitalized. In our study, $42.4 \%$ of subjects $(95 / 224)$ were having one or both problems, either loss of taste or loss of smell on objective evaluation; butut in the studies by Bhattarchjee et al., $(82.0 \%)$ and Bidkar et al., (81.6\%) more than four fifth of study subjects were having anosmia evaluated using objective tests $[25,26]$.

The statistical analysis of the differences in OTD between selected subpopulations, has revealed some interesting points which should be confirmed by studies with larger sample size and multicentric in nature. Although not found statistically significant might be due to small sample size, but older subjects, and co-morbid subjects had lower incidence of loss of taste and smell. Symptoms such as fever, sore throat, shortness of breath, and days of onset of symptoms were significantly associated with OTD. Also, no significant association was found between the extent of the OTD and the severity of the COVID-19 and this evidence should be confirmed by studies with larger sample size, which may reveal the fascinating pathogenetic scenarios. However, considering that there is a correlation between the severity of the systemic clinical picture and viral load on RT-PCR, other local factors are likely to participate in causing the onset of OTD in COVID-19 patients, prospective studies with objective evaluation will be necessary to clarify the prognostic value of OTD [23].

\section{Limitations}

The evaluation for OTD was performed at an advanced period of the clinical course on average around 12.3 days from the onset of clinical symptoms, which can be considered as limitation of the study. Also, the study was single centric, so, authors suggest that upcoming studies to involve more than one centre to enhance generalizability of the observations and to enrol subjects at an early period of clinical course which would be very useful to evaluate the olfactory and gustatory functions when they are mostly affected. Being hospital based study, the true prevalence of hypogeusia/ageusia and hyposmia/anosmia among the COVID-19 positive patient cannot be commented from the present study and the constraints which restricted authors to objectively evaluate the prevalence of loss of smell/taste at the level of community was explored from the pilot study being performed and it included factors such as being tribal area with lower literacy rate (below 50\%), the questionnaire in the local language could not be sent over the social media platform (WhatsApp, Telegram); a lower response rate was observed due to fear to get admitted to hospital while collecting data telephonically or via home visits; and most importantly there was lack of trained field level health care workers to perform the objective tests for hypogeusia/ageusia and hyposmia/anosmia for home isolated COVID-19 patients.

\section{Conclusion}

The present study evaluated the OTD objectively using validated tool kits and it was observed that $42.4 \%$ of subjects (95/224) had olfactory and taste dysfunctions. Taste and smell are among the important senses and should not be taken for granted. Though COVID-19 can present asymptomatically, there are a vast number of patients who present with anosmia and ageusia. In India, generally the COVID-19 induced OTD is noted based on self-reporting without objective evaluation, which results in under reporting of these symptoms. So, an objective evaluation of OTD should be taken into consideration to evaluate OTD using gold standard methods.

\section{Declarations}

Conflict of interest The authors declare that they do not have any conflict of interest.

\section{References}

1. Coronavirus disease (COVID-19) Dashboard [Internet].2021 [cited on 05 February 2021] Available from: https://covid19.who.int.

2. Pormohammad A, Ghorbani S, Khatami A, Farzi R, Baradaran B, Turner DL et al (2020) Comparison of confirmed COVID-19 with SARS and MERS cases - Clinical characteristics, laboratory findings, radiographic signs and outcomes: A systematic review and meta-analysis. Rev Med Virol 30(4):e2112

3. Alimohamadi Y, Taghdir M, Sepandi M (2020) Estimate of the basic reproduction number for COVID-19: A systematic review and meta-analysis. J Prev Med Public Health 53(3):151-157

4. COVID-19 Coronavirus Pandemic [Internet].2021 [cited on 05 February 2021] Available from: https://www.worldometers.info/coronavirus.

5. Xydakis MS, Dehgani-Mobaraki P, Holbrook EH, Geisthoff UW, Bauer C, Hautefort C et al (2020) Smell and taste dysfunction in patients with COVID-19. Lancet Infect Dis 20:1015-1016

6. Symptoms of coronavirus [Internet].2021 [cited on 05 February 2021] Available from: https://www.cdc.gov/coronavirus/2019-ncov/symptoms-testing/ symptoms.html. 
7. Struyf T, Deeks JJ, Dinnes J, Takwoingi Y, Davenport C, Leeflang MM et al. 2020 Signs and symptoms to determine if a patient presenting in primary care or hospital outpatient settings has COVID 19 disease. Cochrane Database Syst Rev 7(7): CD013665

8. Aanand P, Angral S, Varshney S, Raj R (2021) Incidence of Anosmia among Covid 19 patients in India. Indian J Otolaryngol Head Neck Surg. https://doi.org/10.1007/s12070-021-02641-6

9. Rajkumar I, Anand KH, Revathishree K, Shoba K, Srinivasan K (2020) Contemporary Analysis of Olfactory Dysfunction in Mild to Moderate Covid 19 Patients in A Tertiary Health Care Centre. Indian J Otolaryngol Head Neck Surg. https://doi.org/10.1007/s12070-020-02175-3

10. Cain WS, Gent JF, Goodspeed RB, Leonard G (1988) Evaluation of olfactory dysfunction in the Connecticut Chemosensory Clinical Research Center. Laryngoscope 98:83-88

11. Loewen IJ, Boliek CA, Harris J, Seikaly H, Rieger JM (2010) Oral sensation and function: a comparison of patients with innervated radial forearm free flap reconstruction to healthy matched controls. Head Neck 32:85-95

12. Luers JC, Rokohl AC, Loreck N, Wawer Matos PA, Augustin M, Dewald F et al (2020) Olfactory and Gustatory Dysfunction in Coronavirus Disease 2019 (COVID-19). Clin Infect Dis 71(16):2262-2264

13. Roland LT, Gurrola JG II, Loftus PA, Cheung SW, Chang JL (2020) Smell and taste symptom-based predictive model for COVID-19 diagnosis. Int Forum Allergy Rhinol 10(7):832-838

14. De Maria A, Varese P, Dentone C, Barisione E, Bassetti M (2020) High prevalence of olfactory and taste disorder during SARS-CoV-2 infection in outpatients. $J$ Med Virol 92(11):2310-2311

15. Tong JY, Wong A, Zhu D, Fastenberg JH, Tham T (2020) The prevalence of olfactory and gustatory dysfunction in COVID-19 patients: a systematic review and meta-analysis. Otolaryngol Head Neck Surg 63:3-11

16. Mishra P, Gowda V, Dixit S, Kaushik M (2020) Prevalence of new onset anosmia in COVID-19 patients: is the trend different between European and Indian population? Indian J Otolaryngol Head Neck Surg 72(4):484-487

17. Jain A, Kumar L, Kaur J, Baisla T, Goyal P, Pandey AK et al (2020) Olfactory and taste dysfunction in coronavirus disease 2019 patients: its prevalence and outcomes. J Laryngol Otol. https://doi.org/10.1017/S0022215120002467
18. Panda S, Mohamed A, Sikka K, Kanodia A, Sakthivel P, Thakar A et al (2020) Otolaryngologic manifestation and long-term outcome in mild COVID-19: experience from a tertiary care centre in India. Indian J Otolaryngol Head Neck Surg 73(1):1-6

19. Krishnasamy N, Natarajan M, Ramachandran A, Vivian Thangaraj JW, Etherajan T, Rengarajan J et al (2021) Clinical Outcomes among asymptomatic or mildly symptomatic COVID-19 patients in an isolation facility in Chennai. India Am J Trop Med Hyg 104(1):85-90

20. Varghese B, Shajahan S, Anilkumar H, Haridasan RK, Rahul A, Thazhathedath H et al (2020) Symptomatology and epidemiologic characteristics of COVID 19 patients in Kerala. India J Evolution Med Dent Sci 9(46):3411-3417

21. Koul D, Begh RA, Kalsotra P (2021) Olfactory and gustatory alterations in Covid-19 Patients: a tertiary care Covid-19 centre inpatient experience. Indian J Otolaryngol Head Neck Surg. https://doi.org/10.1007/s12070-021-02397-z

22. Thakur K, Sagayaraj A, Prasad KC, Gupta A (2021) Olfactory dysfunction in COVID-19 patients: findings from a tertiary rural centre. Indian J Otolaryngol Head Neck Surg. https://doi.org/10.1007/s12070-021-02364-8

23. Yoon JG, Yoon J, Song JY, Yoon SY, Lim CS, Seong H et al (2020) Clinical significance of a high SARS-CoV-2 viral load in the saliva. J Korean Med Sci 35(20):e195

24. Keyhan SO, Fallahi HR, Cheshmi B (2020) Dysosmia and dysgeusia due to the 2019 Novel Coronavirus: a hypothesis that needs further investigation. Maxillofac Plast Reconstr Surg 42(1):9

25. Bhattacharjee AS, Joshi SV, Naik S, Sangle S, Abraham NM (2020) Quantitative assessment of olfactory dysfunction accurately detects asymptomatic COVID-19 carriers. EClinicalMedicine 28:100575

26. Bidkar V, Mishra M, Selvaraj K, Joshi P, Srikrishna BH, Dabhekar S et al 2020 Testing olfactory and gustatory dysfunctions among quarantine COVID19 suspects. Indian J Otolaryngol Head Neck Surg. Doi; https://doi.org/10.1007/s12070-020-02210-3

Publisher's Note Springer Nature remains neutral with regard to jurisdictional claims in published maps and institutional affiliations. 